\section{AB0330 RELATIONSHIP BETWEEN FOREFOOT SYNOVITIS IN RHEUMATOID ARTHRITIS AND WORSENING FOREFOOT DEFORMITY}

Takeshi Kashiwagura $^{1}$, Moto Kobayashi ${ }^{2}$, Masakazu Urayama ${ }^{3}$, Natsuo Konishi ${ }^{4}$, Hiroki Itoh ${ }^{5}$, Takayuki Tani ${ }^{6}$, Toshiaki Aizawa ${ }^{7}$, Hidekazu Abe $^{8}$, Norio Suzuki ${ }^{9}$, $K_{\text {Keiji Kamo }}{ }^{10}$, Hiroshi Aonuma ${ }^{3,3}$, Yusuke Sugimura ${ }^{11}$, Tetsuya Kawano ${ }^{7,12}$, Yosuke Iwamoto $^{12}$, Tsutomu Sakuraba $^{2}$, Takanori Miura ${ }^{6}$, Manabu Akagawa ${ }^{1}$, Ikuko Wakabayashi ${ }^{1}$, Yoshiaki Kimura ${ }^{1}$, Naohisa Miyakoshi ${ }^{12}$, Yoichi Shimada ${ }^{12}$ ${ }^{1}$ Akita City Hospital, Akita, Japan; ${ }^{2}$ Hiraka General Hospital, Yokote, Japan; ${ }^{3}$ Ogachi Central Hospital, Yuzawa, Japan; ${ }^{4}$ Akita Kousei Medical Center, Akita, Japan; ${ }^{5}$ Noshiro Kousei Medical Center, Noshiro, Japan; ${ }^{6}$ Kakunodate Municipal Hospital, Senboku, Japan; ${ }^{7}$ Kita Akita Municipal Hospital, Kita Akita, Japan; ${ }^{8}$ Ugo Municipal Hospital, Ugo, Japan; ${ }^{9}$ Yuri Kumiai General Hospital, Yuri Honjo, Japan; ${ }^{10}$ Akita Rosai Hospital, Odate, Japan; ${ }^{11}$ Nakadori General Hospital, Akita, Japan; ${ }^{12}$ Akita University Graduate School of Medicine, Akita, Japan

Background: While the number of rheumatoid arthritis (RA) surgeries has been declining due to advances in pharmacotherapies for RA, forefoot surgeries are on the rise. In recent years, the common use of joint ultrasonography in RA consultations has led to the early detection of synovitis. However, little is known about how much forefoot deformities such as hallux valgus and metatarsophalangeal (MTP) joint dislocation are affected by synovitis in the forefoot of RA patients.

Objectives: The present study examined factors involved in forefoot deformity among patients with foot synovitis identified on joint ultrasound. Methods: Subjects (71 patients, 91 feet) were RA patients who had undergone foot joint ultrasonography more than 2 years earlier and underwent standing X-rays of the feet before and after ultrasonography. Surgery cases were excluded. Mean age was 64.9 years (range, 15-90 years). Disease stage was Stage 1 in 14 patients, Stage 2 in 14 patients, Stage 3 in 16 patients, and Stage 4 in 27 patients. According to the Steinbrocker functional classification, RA was Class 1 in 45 patients, Class 2 in 19 patients, Class 3 in 6 patients and Class 4 in 1 patient. Twenty-five patients had been administered biological drugs. At the time of joint ultrasonography, patients were questioned regarding whether they had any complaints involving the forefoot, midfoot or hindfoot (noted separately). The following scans were performed: forefoot (MTP joints 1-5); midfoot (calcaneocuboid, calcaneocuboid and cuneonavicular joints) and hindfoot (peroneal muscle tendon, talocrural joint, talocalcaneal joint and posterior tibial muscle tendon). Foot deformity score (FDS) (hallux valgus angle + first-second intermetatarsal angle $\left[M_{1} M_{2}\right.$ angle] + first-fifth intermetatarsal angle $\left[\mathrm{M}_{1} \mathrm{M}_{5}\right.$ angle]) was used as the benchmark for forefoot deformity, and an increase $>5^{\circ}$ was considered to indicate worsening deformity.

Results: Forefoot deformity had progressed in 25 patients. Mid- and hindfoot synovitis and the presence of complaints were not associated with deformity. However, significant worsening of FDS was observed in patients with forefoot synovitis or forefoot complaints. While no difference in age, disease activity, biologic disease-modifying antirheumatic drug usage or health assessment questionnaire results were seen in the advanced deformity group, duration of disease was significantly shorter in this group.

Conclusion: Mid- and hindfoot synovitis was unrelated to forefoot deformity. MTP joint synovitis in the forefoot was related to forefoot deformity. Continuous synovitis in the forefoot must damage the articular capsule and ligament structure, leading to progression of deformity. As shown by the short duration of the disease in the advanced deformity group, deformity may progress in the early stages among patients with forefoot deformity.

Disclosure of Interests: None declared

DOI: 10.1136/annrheumdis-2019-eular.4112

\section{AB0331 DEPRESSION AND ANXIETY IN PATIENTS WITH RHEUMATOID ARTHRITIS}

Wanruchada Katchamart ${ }^{1}$, Pongthorn Narongroeknawin ${ }^{2}$, Wanwisa Chanapai ${ }^{3}$, Phakhamon Thaweeratthakul'. ${ }^{1}$ Division of Rheumatology, Department of Medicine, Faculty of Medicine Siriraj Hospital, Mahidol University, Bangkok, Thailand, Bangkok, Thailand; ' ${ }^{2}$ Division of Rheumatology, Department of Medicine, Phramongkutklao Hospital and College of Medicine, Bangkok, Thailand, Bangkok, Thailand; ${ }^{3}$ Division of Clinical Trials, Research Department, Faculty of Medicine Siriraj Hospital, Mahidol University, Bangkok, Thailand, Bangkok, Thailand

Background: Rheumatoid arthritis (RA) is a chronic inflammatory joint disease leading to substantial morbidity and mortality and had a major economic and psychological impact on individuals with RA and their families. The prevalence of depressive disorder in RA was $17 \%$ and associated with poorer outcomes
Objectives: To evaluate prevalence of depression and anxiety and their related factors in patients with RA.

Methods: In this cross-sectional study, 464 patients with RA from two RA registries, the Siriraj Rheumatoid Arthritis (SiRA) registry and the Thai Army Rheumatoid Arthritis Cohort (TARAC) were enrolled. Demographic data and clinical variables, including disease activity, functional status, health-related quality of life, and cognitive function, were collected. Depression and anxiety were assessed using the Thai version of the Hospital Anxiety and Depression Scale (Thai HADS).

Results: Based on the Thai HADS cutoff value of more than 8 out of $21,12.5 \%$ and $14.5 \%$ had some degrees of depression and anxiety, respectively. The proportion of depression and anxiety elevated with increasing disease activity or worsening functional status. However, in multivariate analyses, only global health $[\mathrm{RR}(95 \% \mathrm{Cl}) 0.98(0.96-0.99)$, p 0.001] was negatively associated with depression, after adjusted for covariate. For anxiety, functional disability $[\mathrm{RR} \quad(95 \% \mathrm{Cl}) \quad 2.46 \quad(1.33$ 4.54), $\mathrm{p}$ 0.004] and marital status [married: RR $(95 \% \mathrm{Cl}) 2.43$ (1.25 4.73), $p$ 0.009] significantly increased the risk, while disease duration 10 years or more $[R R(95 \% \mathrm{Cl}) 0.45(0.25-0.80), p$ 0.007] and global health [RR $(95 \% \mathrm{Cl}) 0.97(0.95-0.98), \mathrm{p}<0.001]$ decreased this risk. Conclusion: Depression and anxiety are common in patients with RA. Patients' perception of their current health is significantly related to mood disorders. Therefore, mental health status, especially mood disturbances, should be addressed in routine practice to improve quality of life in RA.

\begin{tabular}{lllll}
\multicolumn{3}{l}{ Table 1 Multivariate analyses for depression and anxiety } \\
\hline Factors & \multicolumn{2}{c}{ Depression } & Anxiety \\
\cline { 2 - 5 } & $\mathrm{RR}(95 \% \mathrm{Cl})$ & $\mathrm{P}$ value & $\mathrm{RR}(95 \% \mathrm{Cl})$ & $\mathrm{P}$ value \\
\hline $\mathrm{DAS} 28>2.6$ & $1.66(0.49-5.70)$ & 0.418 & & \\
$\mathrm{HAQ}>0.5$ & $1.38(0.75-2.54)$ & 0.303 & $2.46(1.33-4.54)$ & 0.004 \\
Comorbidity & $1.04(0.58-1.89)$ & 0.890 & $1.05(0.59-1.86)$ & 0.875 \\
Global health & $0.98(0.96-0.99)$ & 0.001 & $0.97(0.95-0.98)$ & $<0.001$ \\
Cognitive impairment & $1.12(0.59-2.15)$ & 0.727 & $1.66(0.84-3.30)$ & 0.144 \\
Pain score & $1.07(0.95-1.20)$ & 0.275 & $1.01(0.90-1.14)$ & 0.819 \\
Disease duration $\geq 10$ years & & & $0.45(0.25-0.80)$ & 0.007 \\
Marital status (married) & & & $2.43(1.25-4.73)$ & 0.009 \\
\hline
\end{tabular}

Disclosure of Interests: None declared

DOI: 10.1136/annrheumdis-2019-eular.3169

\section{AB0332 CLINICAL FEATURES AND RISK FACTORS OF CHRONIC HEART FAILURE IN PATIENTS WITH EARLY RHEUMATOID ARTHRITIS PRIOR TO THERAPY WITH BASIC ANTI-INFLAMMATORY DRUGS}

Irina Kirillova, Diana Novikova, Tatiana Popkova, Eugenia Markelova, Helen Udachkina, Yulia Gorbunova, Maria Cherkasova. V.A. NASONOVA RESEARCH INSTITUTE OF RHEUMATOLOGY, Moscow, Russian Federation

Background: Heart failure (HF) is a major cause of premature mortality, there is little information regarding its prevalence and associated risk factors in patients (pts) with early rheumatoid arthritis (RA).

Objectives: to study the frequency, clinical manifestations and risk factors associated with the development of HF in pts with early RA prior to therapy with basic anti-inflammatory drugs.

Methods: A total of 74 pts with early RA (ACR/EULAR criteria, 2010) were included in the study: $78 \%$ of women, median (Me) age - 56 years [47; 61], Me of disease duration - 7 [4;8] months; Me DAS28 5.3 [5.0;6.2], IgM RF seropositive $(87 \%)$ and/or ACCP $(100 \%)$, without any experience of administration of diseasemodifying antirheumatic drugs and glucocorticoides. All pts underwent blood pressure monitoring, echocardiography, tissue Doppler imaging, NT-proBNP. The normal range for NT-proBNP was less than $125 \mathrm{pg} / \mathrm{ml}$. HF was diagnosed according to the recommendations of the European Society of Cardiology (2012).

Results: HF was diagnosed in 24 (33\%) pts: in 23 pts - HF with preserved ejection function (EF), in 1 pts - HF with reduced EF. Dyspnea was detected in 21 (87\%) RA pts with HF (positive predictive value (PPV)-33\%), in 6 (25\%) - ankle edema (PPV-35\%), in $24(100 \%)$ fatigue (PPV-38\%). In $5(21 \%)$ pts dyspnea $\mathrm{NYHA}=1$ was observed, 15 $(63 \%)-\mathrm{NYHA}=2$, in $1(4 \%)-\mathrm{NYHA}=3$. Diastolic dysfunction of the left ventricle was detected in all pts with HF (PPV-69\%). Elevated NTproBNP level wasn't highly indicative for HF in early RA (PPV=41\%). All pts with early RA were divided into 2 groups: 1- with HF, 2-without HF. Pts with RA and HF were older $(61[58 ; 65]$ vs $51[36 ; 56]$ years $)$, had higher BMI $\left(28[25 ; 32]\right.$ vs $\left.24[22 ; 29] \mathrm{kg} / \mathrm{m}^{2}, \quad \mathrm{p}<0,05\right)$, NT-proBNP level $(192.0$ [154.9;255.7] vs $77.0[41.1 ; 191.2] \mathrm{pg} / \mathrm{ml}, \quad \mathrm{p}<0.05)$, more likely to have arterial hypertension $(\mathrm{AH})(83 \%$ vs $51 \%, \mathrm{p}<0.05)$, carotid atherosclerosis $(91 \%$ vs $48 \%, p<0.05)$, coronary artery disease (CAD) $(40 \%$ vs 